\section{Heat Transfer Between a Non-Wetted Surface and a Moving Fluid}

RECENT papers on heat transfer to liquid metals have raised the question whether different rates of heat transfer are obtained from wetted and nonwetted surfaces when the surface temperature is below the boiling point of the fluid. To my knowledge, no paper has presented data comparing the wetted and non-wetted cases.

An experimentel study of heat transfer to mercury in circular tubes and annuli is being made. Heating and cooling results have been obtained with nonwetted stainless steel and with completely wetted and amalgamated copper tubes. The range of Reynolds number, based on four times the hydraulic radius, was from 5,000 to 100,000 . The Prandtl number was about $0 \cdot 02$. Although less accurate for obtaining absolute values, the apparatus is accurate to \pm 2 per cent when comparing heat transfer coefficients. Within the limitations of the apparatus, the results for both wetted and non-wetted tubes were identical.

It would appear that the nature of contact between a liquid and a solid does not affect heat transfer across the interface. This suggests hydrodynamic identity of the wetted and non-wetted cases, and supports the generally accepted conclusion, drawn from friction tests, that there is no slip at a liquid-solid interface, even when wetting does not occur.

This investigation is sponsored by the Ministry of Supply.

\section{LLOYD M. TREFETHEN}

Department of Engineering, University of Cambridge. Jan. 31

\section{Selective Absorption of Sulphur-35 by Fungus-infected Leaves}

IT has been shown that fungi and higher plants absorb and accumulate fungicidal substances to a high degree $e^{1-3}$; but, so far as we are aware, no quantitative demonstration of selective absorption of therapeutic chemicals by parasitized tissues of plants or animals has been reported. The successful therapeutic use of sulphur vapours against external powdery mildews and internal rusts ${ }^{4}$ suggested that these infections would be suitable for the study of selective absorption of a fungicide.

Bean leaves infected with the uredinial stage of rust (Uromyces phaseoli) or with powdery mildew (Erysiphe polygoni), and sunflower leaves infected with rust (Puccinia helianthi), were detached from living greenhouse plants and exposed to the vapours from $50 \mathrm{ml}$. of 0.01 per cent sodium sulphide $\left(\mathrm{Na}_{2} \mathrm{~S}_{2} 9 \mathrm{H}_{2} \mathrm{O}\right)$ containing 5-8 microcuries of sulphur-35 (halflife $87 \cdot 1$ days, $0 \cdot 169-\mathrm{MeV} . \beta^{-}$, supplied by U.S. Atomic Energy Commission, Oak Ridge, Tennessee) at $p \mathrm{H} 8$ in sealed pint jars for $8 \mathrm{hr}$. at about $22^{\circ} \mathrm{C}$. This treatment was lethal to the rust or powdery mildew mycelium in or on the leaves, but did not injure the host. The sulphur-35 served only as a tracer, as dilute hydrogen sulphide gas, vapours from commercial lime sulphur, and from the sodium sulphide without sulphur-35 have been therapeutically effective.
The localization of sulphur in the leaves was determined by making radioautographs of treated leaves on X-ray film. An exposure period of $48 \mathrm{hr}$. was satisfactory. Radioautographs of bean leaves inoculated with rust four to twenty-one days before treatment showed greater uptake of sulphur by infected than by non-infected areas of the same leaf or by healthy leaves. For leaves with infections eight days old (Figs. $A$ and $B$ ) and with fungus colonies (uredinial pustules) about $1.2 \mathrm{~mm}$. in diameter in one representative test, most lesions showed a centre of strong radiation of about $0.5 \mathrm{~mm}$. in diameter, encircled by zones of about $1.0,1.8$ and $3.5 \mathrm{~mm}$. in which the radiation was weaker, stronger, and weaker, respectively, than in the zones immediately within. The outer (weaker) zone, well beyond the limit of the rust mycelium, showed less radiation than those portions of the leaf beyond the apparent influence of the mycelium. In one case radioautographs of leaves treated four days after inoculation showed the location of the pustules before they were visible to the unaided eye. In general, the older and larger the pustules the more intense the radioautograph (Fig. $C$ ), but in several cases the radioautographs showed much weaker radiation at the centres of the pustules than from areas beyond the centres. The radioautographs indicate greater sulphur absorption by infected than by healthy tissues for the three diseases studied.

When the sulphur-35 radiation from bean leaves was localized to areas of $0.2-13 \mathrm{~mm} .^{2}$ by means of round holes in lead shields and measured in a Geiger counter, the radiation from mildewed and rusted areas was up to five times that of normal areas of the same leaves. The amount of absorbed sulphur necessary for destroying rust and mildew infections without injury to the hast varied greatly, but was of the order of 1-3 $\mu \mathrm{gm}$. sulphur per cm. ${ }^{2}$ of leaf area, while the amount absorbed where slight host injury occurred was of the order of 3-6 $\mu \mathrm{gm}$. While the range between therapy and host injury seems small in terms of the amount of sulphur absorbed, it was large in terms of the amount of sulphur applied, as a ten-fold increase in dosage resulted

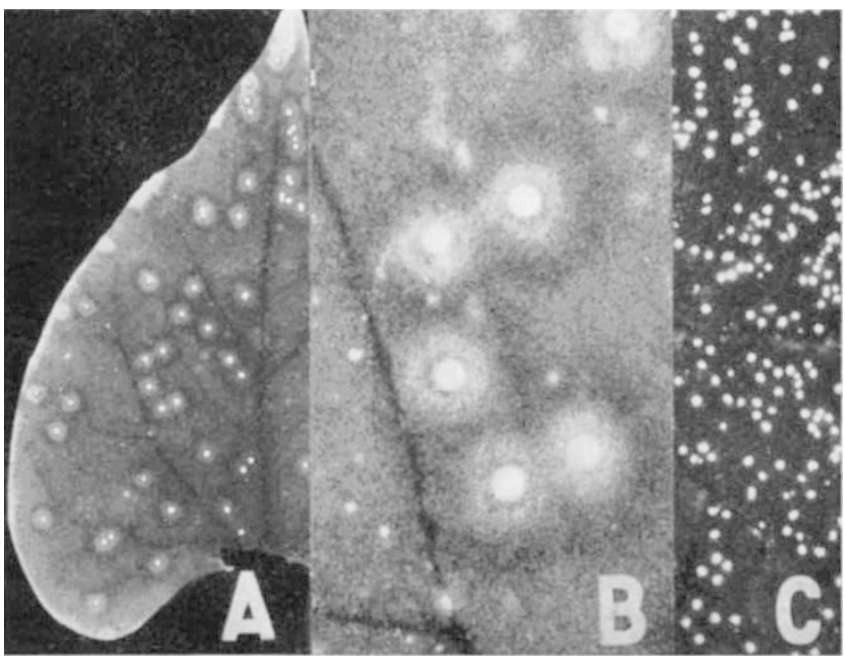

A. Positive print of radioautograph of half of a rust-infected bean leaf treated with sulphur-35 eight days after inoculation. The target-shaped areas are the positions of the uredinial pustules. Actual size. B. Enlargement or portion of $A$. $C$. Print of radioautograph of portion of bean leaf treated twelve days after inoculation. Actual size. In all cases the light areas represent radioactivity 